\title{
Justification of dead zone presence in catalyst pellets.
}

\author{
Mirosław Szukiewicz, Elżbieta Chmiel-Szukiewicz, Krzysztof.Kaczmarski, Adrian Szałek
}

Department of Chemical and Process Engineering, Rzeszow University of Technology, POLAND

35-959 Rzeszów, al. Powstańców Warszawy 6,

E-mail:ichms@prz.edu.pl

\begin{abstract}
Heterogeneous catalytic processes and biocatalitic processes are the most important types of the processes where existence of zones without reactants were reported. The aim of the work is justification of presence of dead zone in the catalyst pellet by experimental verification of a model solution. Good consistency of theretical and experimental results justify that dead zone is present inside the catalyst pellet.
\end{abstract}

Keywords - heterogeneous catalysis, dead zone, dead core, effectiveness factor, diffusional resistances

\section{Introduction}

Heterogeneous catalytic processes and biocatalitic processes are the most important types of the processes where existence of zones without reactants were reported. And we don't think about physical defects of catalysts resulted mainly from imperfections of manufacturing or activation processes regions of catalysts or biofilms without reactants that arise spontaneously as a result of fast reaction run, despite the fact that in this area catalyst or bio-catalyst (enzyme) could be active. The inactive zone lessens reactor or bioreactor productivity and influences on process economy -it can reduce or even destroy in large scale processes effects obtained in laboratory. For heterogeneous catalysis the zone without reactants was called "dead zone" and it was predicted by Aris[1]. The analytic solution of non-linear problem without external mass transfer limitations and without the dead zone was presented by Mehta and Aris [2] while for the presence of dead zone by York et al. [3]. Szukiewicz [4] extends applicability region of these solutions on the case of important external mass transfer resistances. The aim of the work is justification of presence of dead zone in the catalyst pellet by experimental verification of a model solution.

\section{Results and Discussion}

Catalytic micro-reactor (the Microactivity Effi reactor, PID Eng\&Tech, Spain) of diameter $9.1 \mathrm{~mm}$ equipped with hydrogen generator, was used for investigations. In the reactor was carried out hydrogenation of propylene. As a hydrogenation catalyst the KUB-3 catalyst (the main components of the catalyst are $\mathrm{Ni}, \mathrm{NiO}$, and $\mathrm{Al}_{2} \mathrm{O}_{3}$.) manufactured by INS, Puławy, Poland was chosen. Catalyst form: plate with diameter 4-6 $\mathrm{mm}$ and thicknes $0.4 \mathrm{~mm}$. Experiments were carried out at temperature range $100-150^{\circ} \mathrm{C}$ under pressure $1.2 \times 10^{5} \mathrm{~Pa}$.

On the basis of experiments carried out the following kinetic equation was developed:

$$
r_{p}=44400 \mathrm{p}_{\mathrm{p}}^{0.5} \mathrm{e}^{\frac{-26500}{\mathrm{RT}}} \quad\left[\mathrm{mol} /\left(m^{3} \mathrm{~s}\right)\right](1)
$$

The kinetic equation indicates that inside the catalyst pellet a dead zone can be formed. Consequently, a mathematical model of the process includes typical mass balance equation with boundary conditions characteristic for dead zone problem [1]. The analytic solution for the problem under consideration is given by:

$$
c=\left(\frac{1-n}{2} \sqrt{\frac{2 \Phi^{2}}{1+n}}\right)^{2 /(1-n)}\left(x-x_{d z}\right)^{2 /(1-n)}
$$


where $\Phi$ is Thiele modulus, $\mathrm{n}$ is an exponent in the kinetic equation. $\mathrm{x}_{\mathrm{dz}}$ (coordinate that determines dead zone location) and $\mathrm{c}_{\mathrm{s}}$ (surface concentration) can be determined from:

$$
1-x_{d z}=\frac{2}{1-n} \sqrt{\frac{1+n}{2 \Phi^{2}}} c_{s}{ }^{(1-n) / 2} \text { and } \sqrt{\frac{2 \Phi^{2}}{1+n}} c_{s}{ }^{(1+n) / 2}=B i_{m}\left(1-c_{s}\right)
$$

Effectiveness factor is given by

$$
\eta=\sqrt{\frac{2 c_{s}{ }^{1+n}}{(1+n) \Phi^{2}}}
$$

Effectiveness factor values calculated theoretically and determined from experiment were compared. Results are presented in Table 1. The calculated and determined experimentally values of effectivess factor have close values. The error does not exceed 10\% (with one exception - E9) so consistency of results can be treated as good. Determined size of dead zone was about $75 \%$, so a greater part of catalyst pellet remains inactive.

Table 1 Experimental and theoretical values of effectiveness factor

\begin{tabular}{|c|c|c|c|c|c|c|}
\hline $\begin{array}{c}\text { Exp. } \\
\text { number }\end{array}$ & $\begin{array}{c}\text { Molar } \\
\text { ratio }\end{array}$ & $\begin{array}{c}\text { Temp. } \\
{\left[{ }^{\circ} \mathrm{C}\right]}\end{array}$ & $\begin{array}{c}\mathrm{D}_{\mathrm{ef}} \cdot 10^{7} \\
{\left[\mathrm{~m}^{2} / \mathrm{s}\right]}\end{array}$ & $\mathrm{k}_{\mathrm{g}}$ & $\eta_{\exp }$ & $\eta_{\text {calc }}$ \\
\hline E1 & 0.10 & 101.4 & 2.95 & 0.178 & 0.0709 & 0.0780 \\
\hline E2 & 0.14 & 111.2 & 3.58 & 0.210 & 0.0669 & 0.0828 \\
\hline E3 & 0.12 & 111.3 & 3.58 & 0.196 & 0.0759 & 0.0794 \\
\hline E4 & 0.14 & 120.3 & 3.81 & 0.214 & 0.0856 & 0.0765 \\
\hline E5 & 0.16 & 120.0 & 3.81 & 0.228 & 0.0840 & 0.0796 \\
\hline E6 & 0.20 & 130.0 & 3.73 & 0.424 & 0.0840 & 0.0752 \\
\hline E7 & 0.14 & 142.0 & 3.79 & 0.275 & 0.0919 & 0.0925 \\
\hline E8 & 0.20 & 141.5 & 3.79 & 0.327 & 0.0986 & 0.1024 \\
\hline E9 & 0.20 & 151.0 & 3.94 & 0.439 & 0.0758 & 0.0618 \\
\hline
\end{tabular}

\section{Conclusion}

Good consistency of theretical and experimental results justify that dead zone is present inside the catalyst pellet since presented in References regular and dead zone model are complementary.

\section{Acknowledgments}

The financial support of the National Science Centre, Poland (Project 2015/17/B/ST8/03369) is gratefully acknowledged.

\section{References}

[1] R. Aris. J. K. Author, "The Mathematical Theory of Diffusion and Reaction in Permeable Catalysts. Vol I. The Theory of the Steady State" Oxford: Clarendon Press, 1975.

[2] B.N. Mehta, R. Aris, "A Note on a Form of the Emden-Fowler Equation” J. Math. Anal. Appl., vol. 36, pp. 611-621, 1971.

[3] R.L. York, K.M. Bratlie, R.L. Hile, L.K. Jang, "Dead zones in porous catalysts: Concentration profiles and efficiency factors", Catalysis Today, vol. 160, pp. 204-212, 2011.

[4] M. K. Szukiewicz, "Exact analytical solution of a non-linear reaction-diffusion problem for full range of parameters values- multiplicity and dead zone coexistence." Mathematics in (bio)Chemical Kinetics and Engineering MaCKiE-2015, Ghent, 2015, pp. 24-26. 